\title{
Sudden decay of indirect exchange coupling between magnetic atoms on carbon nanotubes
}

\author{
D. F. Kirwan, ${ }^{1}$ C. G. Rocha, ${ }^{1}$ A. T. Costa, ${ }^{2}$ and M. S. Ferreira ${ }^{1, *}$ \\ ${ }^{1}$ School of Physics, Trinity College Dublin, Dublin 2, Ireland \\ ${ }^{2}$ Instituto de Física, Universidade Federal Fluminense, Niterói, Rio de Janeiro 24001-970, Brazil \\ (Received 15 October 2007; revised manuscript received 21 December 2007; published 28 February 2008)

\begin{abstract}
Indirect exchange coupling plays a central role in determining the magnetic order in systems composed of adsorbed magnetic moments on a metallic host. For low-dimensional metallic structures, such as nanotubes, this interaction is predicted to decay rather slowly. Ab initio calculations have nevertheless been unable to reproduce this prediction. To clarify this matter, we make use of a simple analytical expression for the indirect exchange coupling that, on the one hand, confirms the long ranged nature of this interaction, and, on the other hand, points to situations in which the coupling may display unexpectedly shorter ranges. We show that the interaction range depends rather sensitively on the location of the magnetic moments, which explains the difficulty in probing the long range character of the indirect exchange coupling from standard $a b$ initio calculations.
\end{abstract}

DOI: 10.1103/PhysRevB.77.085432

PACS number(s): 75.75.+a, 73.63.Fg

In the continuous search for materials that can sustain our incessant demand for smarter, faster and more efficient computers, the field of magnetoelectronics appears as a promising alternative. Also known as spintronics, this has been a field of research with strong levels of investment over the past decade and with tremendous potential for technological applications. When combined with systems of reduced dimensionality, such as thin films, nanowires, and molecular structures, this field is expected to pave the way to the production of nonvolatile computer memories, extremely efficient magnetic sensors, and magnetic materials with enhanced information storage capacity, to name but a few.

Carbon nanotubes are one prime example of lowdimensional structures that are potentially useful for their magnetotransport properties. The ability to produce sizable changes in the conductance of a nanotube due to an applied magnetic field is one of the driving forces in the research of magnetic properties of carbon-based structures. ${ }^{1,2}$ For that purpose, nanotubes must interact with magnetic foreign objects that lift the intrinsic spin balance of a nonmagnetic material, such as carbon. Substrates, ${ }^{3,4}$ substitutional impurities, ${ }^{5}$ adsorbed atoms, ${ }^{6,7}$ and nanoparticles ${ }^{8}$ are some of the different magnetic foreign objects that can interact with carbon nanotubes. Among those, transition-metal magnetic adatoms have been reported to produce noticeable changes in the spin-dependent electronic structure of carbon nanotubes. ${ }^{6,8}$ Furthermore, the formation of defect-induced magnetic moments in carbon-based materials appears as an additional possibility to manipulate the magnetic response of these systems. ${ }^{9}$

Motivated by the interaction of carbon nanotubes with magnetic objects, we have recently addressed the problem of indirect exchange coupling (IEC) between localized magnetic moments mediated by the conduction electrons of nanotubes. ${ }^{10}$ More specifically, in Ref. 10, we have considered how two magnetic adatoms attached to the walls of a nanotube and separated by a distance $D$ are mutually coupled. Surprisingly, we found that the coupling lacks the typical oscillations observed in indirect interaction of magnetic objects embedded in metallic hosts. We have explained this lack of oscillations as a commensurability effect respon- sible for hiding the truly oscillatory character of the IEC. Furthermore, we have also reported this indirect interaction as being of very long range and argued that this may be further explored to control the response of magnetic doping agents. In particular, the predicted $1 / D$ scaling of the coupling implies that the magnetic moments of adatoms are able to feel each other's presence even when they are very far apart. Such a slow decaying rate appears as a consequence of the intrinsic dimensionality of the nanotube and should, therefore, be common enough to be seen in a variety of combinations of nanotubes and magnetic adatoms. Nevertheless, this ubiquity has not been observed. In fact, first-principles calculations have been unable to reproduce the claimed long ranged interaction experienced by magnetic adatoms on these materials. ${ }^{11}$

It is the goal of this paper to clarify that the IEC between localized moments on nanotubes is indeed long ranged but also to show that there are situations in which the magnetic coupling may decay rather abruptly. We show that the latter is rather common in the case of nanotubes, which explains the difficulties in probing the long range of the coupling by first-principles calculations. In doing so, we also identify that differences in the range of the IEC may give indications about where the magnetic moments are located with respect to the underlying carbon lattice. Combined with the ability to control the IEC through doping, ${ }^{10}$ we argue that changes in the range of the magnetic interaction may be externally controlled. In what follows, we provide a simple expression for the IEC between two adatoms on a nanotube. The simplicity of this expression allows us to trace the dependence of the coupling on the adatom separation, leading us to an analytical expression that confirms the general conclusion that the IEC on one-dimensional hosts does decay rather slowly. Further investigations of this expression point to the cases in which the range of the magnetic interaction may be dramatically reduced.

We consider two magnetic atoms, labeled $A$ and $B$, attached to the walls of an infinitely long carbon nanotube and schematically represented in Fig. 1. Magnetism in these atoms is driven by an intra-atomic Coulomb interaction that, when treated in mean-field approximation through a self- 


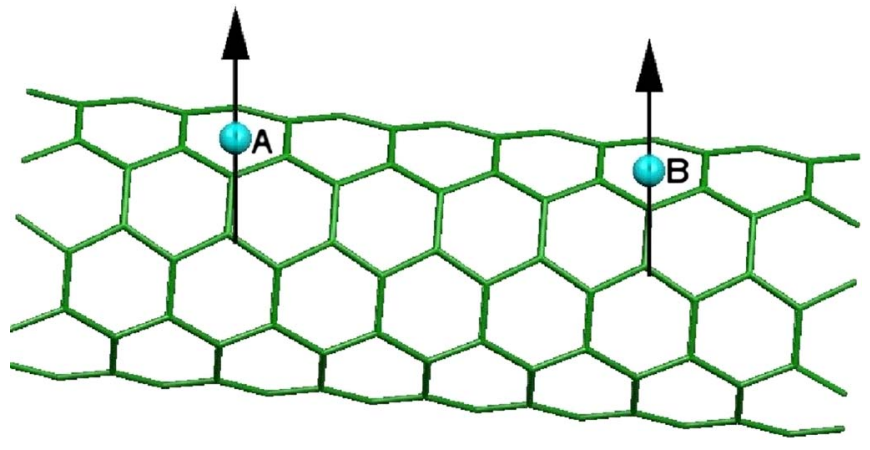

FIG. 1. (Color online) Schemetic representation of an armchair nanotube with two magnetic adatoms. The arrows indicate the direction of their magnetic moments.

consistent procedure, can be described by an effective spindependent potential located at the atomic positions. In this way, the electronic structure of the entire system is well described by a Hamiltonian in a basis of localized atomic orbitals. In such a basis, this tight-binding-like Hamiltonian is fully determined by the on-site potentials and hopping integrals.

We assume that the magnetic moments of the individual adatoms are initially parallel, hereafter referred to as the ferromagnetic configuration (as schematically illustrated in Fig. 1). In this configuration, the Hamiltonian of the entire system written in the basis $|j\rangle$ of atomic orbitals centered at a site $j$ is given by $\hat{H}=\hat{H}_{N T}+\hat{H}_{A}+\hat{H}_{B}+\hat{V}_{C}$, where $\hat{H}_{N T}=\Sigma_{j, j^{\prime}}|j\rangle \gamma\left\langle j^{\prime}\right|$ is the Hamiltonian of the individual nanotube, $\hat{H}_{A}=|A\rangle \epsilon_{A}\langle A|$ and $\hat{H}_{B}=|B\rangle \epsilon_{B}\langle B|$ are the Hamiltonians associated with the atoms $\mathrm{A}$ and $\mathrm{B}$, respectively, and $\hat{V}_{C}=\Sigma_{\ell} t\{|A\rangle\langle\ell|+| \ell\rangle\langle A|\}$ $+\sum_{\ell^{\prime}} t^{\dagger}\left\{|B\rangle\left\langle\ell^{\prime}|+| \ell^{\prime}\right\rangle\langle B|\right\}$ refers to the coupling between the adatoms and the nanotube. The parameters $\gamma, \epsilon_{A}, \epsilon_{B}$, and $t$ are all matrices in spin and orbital spaces and correspond to the hopping between nearest-neighbor sites in the nanotube, the on-site potentials of atoms $\mathrm{A}$ and $\mathrm{B}$, and the hopping between the nanotube atoms and the adatoms, respectively. Likewise, the basis $|j\rangle$ represents vectors in the same linear space. It is evident from the expressions above that sites $j$ $=A$ and $j=B$ label the two adatoms and the indices $\ell$ and $\ell^{\prime}$ label the nanotube sites that are coupled to those respective atoms. As we shall see, depending on the selection of which carbon atoms are connected to the magnetic adatoms, one may obtain totally different results for the indirect exchange coupling.

We define the indirect coupling as the amount of energy required to rotate one magnetic moment relatively to the other by an angle $\pi$. In other words, it is defined as the energy to take the moments from the ferromagnetic configuration to the situation in which both moments are oppositely aligned, also referred to as the antiferromagnetic configuration. The energy required to rotate one of the moments by an angle $\theta$ is given by the following expression: ${ }^{10}$

$$
\begin{aligned}
\Delta \mathcal{E}(\theta)= & \frac{1}{\pi} \int_{-\infty}^{\infty} d \omega\left[\frac{1}{1+e^{\beta(\omega-\mu)}}\right] \operatorname{Im} \operatorname{Tr} \ln \left[1+2 V_{x}^{2}(1\right. \\
& \left.-\cos \theta) G_{A, B}^{\uparrow}(\omega) G_{B, A}^{\downarrow}(\omega)\right],
\end{aligned}
$$

where $\theta$ is the angle of rotation and $V_{x}$ is a matrix in orbital space representing the strength of the local exchange potentials. $G_{m, \ell}^{\sigma}(\omega)$ represents the propagator between sites $j=\ell$ and $j=m$ for electrons of spin $\sigma$ and energy $\omega$ in the ferromagnetic configuration, and the trace is over orbital indices. The fraction within brackets is the Fermi function, where $\beta$ $=1 / k_{B} T, k_{B}$ is the Boltzmann constant, $T$ is the temperature, and $\mu$ is the Fermi energy. The coupling is therefore expressed by $\Delta \mathcal{E}(\pi)$.

For the sake of simplicity, the electronic structure of the system will be treated within the single-band tight-binding model. The expressions above are very general and by no means restricted to such a simple case. The results obtained here can be easily extended to a multiorbital description but bring no qualitative difference. This is justified by the fact that the main features of the indirect exchange coupling are predominantly determined by the extended electrons of the host, in this case the nanotube, whose electronic structure is known to be well reproduced by a single-band tight-binding model. With transition-metal atoms in mind, the adatoms are described by a five-fold degenerate $d$ band with the appropriate occupation to represent typical magnetic materials. In this way, rather than matrices in orbital indices, all quantities in the integrand of Eq. (1) become scalar.

The indirect coupling $\Delta \mathcal{E}(\pi)$ can be calculated by inserting the appropriate Green function matrix elements $G_{A, B}^{\uparrow}(\omega)$ and $G_{B, A}^{\downarrow}(\omega)$ into Eq. (1) and evaluating the corresponding energy integral. Since we are interested in assessing how fast the indirect coupling decays, we must investigate how Eq. (1) depends on the adatom separation $D$. A closer inspection shows that the only $D$ dependence in the expression for the coupling lies in the Green function matrix elements $G_{A, B}^{\uparrow}(\omega)$ and $G_{B, A}^{\downarrow}(\omega)$. This becomes more evident when treating the potential $\hat{V}_{C}$ as a perturbation and using Dyson's equation to express how the Green function matrix elements in question are written in terms of those associated with the magnetic atoms and nanotubes in isolation. It is clear that the $D$ dependence of the coupling is entirely contained within this function. More specifically, the dependence on adatom separation enters as a sum over propagators of an isolated nanotube $\mathcal{G}_{\ell, \ell^{\prime}}$ where the sums $\ell, \ell^{\prime}$ extend over all atoms in contact with adatoms $\mathrm{A}$ and $\mathrm{B}$, respectively. We, therefore, define a function $\Gamma_{a, b}^{(n)}$ such that $\Gamma_{a, b}^{(n)}=t^{2} \sum_{\ell, \ell^{\prime}=1}^{n} \mathcal{G}_{\ell, \ell^{\prime}}$, where $n$ is the number of connections each adatom makes to the nanotube host and $t$ is the hopping integral between the adatom and carbon atom. For a single connection, $\Gamma_{a, b}^{(1)}$ reduces to a single propagator. Note that the integers $\ell$ and $\ell^{\prime}$ label the carbon atoms that are connected to adatoms $\mathrm{A}$ and $\mathrm{B}$, respectively. We can also define similar sums $\Gamma_{a, a}^{(n)}, \Gamma_{b, b}^{(n)}$, which are the corresponding sums over matrix elements linking atoms connected to the same adatom and as such contain no $D$ dependence.

With this definition, the expression for $G_{A, B}^{\sigma}$ can be written as

$$
\begin{aligned}
G_{A, B}^{\sigma}= & g_{A A}^{\sigma} \Gamma_{a, b} g_{B B}^{\sigma}\left(\left(1-g_{A A}^{\sigma} \Gamma_{a, a}\right)\left(1-g_{B B}^{\sigma} \Gamma_{b, b}\right)(1\right. \\
& \left.\left.-\frac{g_{A A}^{\sigma} \Gamma_{a, b} \Gamma_{b, a} g_{B B}^{\sigma}}{\left(1-g_{A A}^{\sigma} \Gamma_{a, a}\right)\left(1-g_{B B}^{\sigma} \Gamma_{b, b}\right)}\right)\right)^{-1} .
\end{aligned}
$$



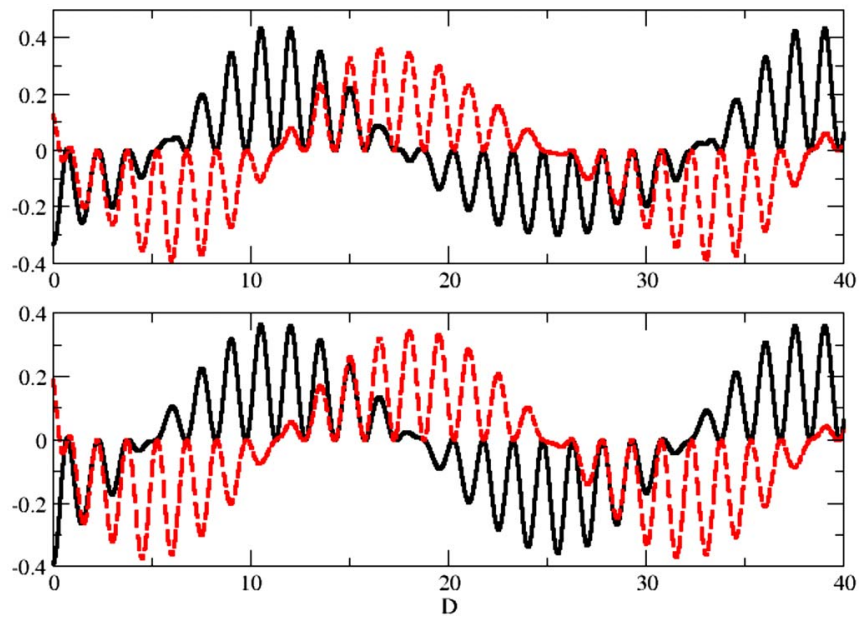

FIG. 2. (Color online) (top) Real (solid line) and imaginary (hatched line) parts of $G_{A, B}^{\uparrow} G_{B, A}^{\downarrow}$ and (bottom) $\operatorname{Tr} \ln (1$ $\left.+4 V_{x}^{2} G_{\mathrm{A}, \mathrm{B}}^{\uparrow} G_{\mathrm{B}, \mathrm{A}}^{\downarrow}\right)$ as a function of the adatom separation $D$ evaluated at $\omega=0.1 \gamma$. Both functions are seen to oscillate with the same periodicity.

The integrand in Eq. (1) can be expected to oscillate as a function of $D$ with the same periodicity as that of the product $G_{A, B}^{\uparrow} G_{B, A}^{\downarrow}$ given by Eq. (2). This is confirmed by Fig. 2, showing the $D$ dependence of both the integrand of Eq. (1) and of the product mentioned above for the case in which each adatom is connected only to a single nanotube site. Both functions vary with the distance $D$ in an oscillatory fashion and have exactly the same periodicities, although the oscillation periods depend on the precise choice of energy $\omega$.

When integrated over energy, the oscillations displayed by the integrand are likely to interfere both destructively and constructively, determining the overall behavior of the indirect coupling as a function of the adatom separation. To prove this point explicitly, we expand part of the integrand (for $\theta=\pi$ ) in a Fourier series of the type

$$
\frac{1}{\pi} \operatorname{Tr} \ln \left[1+4 V_{x}^{2} G_{a, b}^{\uparrow}(\omega) G_{b, a}^{\downarrow}(\omega)\right]=\sum_{m} C_{m}(\omega) e^{i Q_{m}(\omega) D},
$$

leaving us with the following expression for the coupling:

$$
\Delta \mathcal{E}(\pi)=\operatorname{Im} \sum_{m} \int_{-\infty}^{\infty} d \omega\left[\frac{C_{m}(\omega) e^{i Q_{m}(\omega) D}}{1+e^{\beta(\omega-\mu)}}\right] .
$$

$C_{m}(\omega)$ and $Q_{m}(\omega)$ are the Fourier coefficents and corresponding wave vectors of the integrand for a given energy $\omega$. The integral above can be evaluated by assuming that asymptotically $(D \gg 1)$, the dominant contribution comes from the energies surrounding the Fermi energy $\omega=\mu$. This becomes evident when the integral is replaced by a contour integral in the upper half of the complex energy plane. In this case, the poles of the integrand are given by those that vanish the denominator of the Fermi function, the so-called Matsubara frequencies. They are $\omega_{p}=\mu+i(2 p+1) \pi k_{B} T$, where $p$ is an integer that labels the different poles. Given that the Fourier coefficients are slowly varying functions of the energy, $C_{m}(\omega)$ can be replaced with $C_{m}(\mu)$. Moreover, when ex- panded in a Taylor series around $\omega=\mu$, the wave vector $Q_{m}(\omega)$ can be written as $Q_{m}(\omega)=Q_{m}(\mu)+Q_{m}^{\prime}(\omega-\mu)$, where $Q_{m}^{\prime}$ is the energy derivative of $Q_{m}$ evaluated at the Fermi level. Therefore, Eq. (4) may be written as

$$
\Delta \mathcal{E}(\pi)=\operatorname{Im} \sum_{m} C_{m}(\mu) e^{i Q_{m}(\mu) D} \int_{-\infty}^{\infty} d \omega \frac{e^{-Q_{m}^{\prime}(\omega-\mu) D}}{1+e^{\beta(\omega-\mu)}} .
$$

By summing over all the residues, one then finds that the IEC becomes

$$
\Delta \mathcal{E}(\pi)=\operatorname{Im} \sum_{m} \frac{C_{m}(\mu) e^{i Q_{m}(\mu) D} k_{B} T}{\sinh \left(2 k_{B} T Q_{m}^{\prime} \pi D\right)} \approx \operatorname{Im} \sum_{m} \frac{C_{m}(\mu) e^{i Q_{m}(\mu) D}}{2 Q_{m}^{\prime} \pi D},
$$

where the last expression on the far right corresponds to taking the low temperature limit. It is clear that in the asymptotic limit of large values of $D$, each Fourier component $m$ contributes to the IEC with a slowly decaying oscillatory function proportional to $\cos (q D) / D$. Bearing in mind the generality of the arguments used above, Eq. (6) is a convincing result that corroborates our earlier statement that the IEC should display long ranged features and decay rather slowly with the adatom separation. While this statement is true with quite some generality, there are specific cases in which a faster decay is observed. These correspond to the Fourier coefficients, $C_{m}(\mu)$ in Eq. (4) vanishing. If this occurs, to order $p$, say, it becomes necessary to expand the coefficients about $\omega=\mu$ and Eq. (5) becomes

$$
\begin{aligned}
\Delta \mathcal{E}(\pi)= & \operatorname{Im} \sum_{m} \frac{(-1)^{p}}{p !} \frac{d^{p} C_{m}(\mu)}{d \omega^{p}} \frac{e^{i Q_{m}(\mu) D}}{\left[Q_{m}^{\prime}\right]^{p}} \\
& \times \frac{d^{P}}{d D^{P}}\left\{\int_{-\infty}^{\infty} d \omega \frac{e^{-Q_{m}^{\prime}(\omega-\mu) D}}{1+e^{\beta(\omega-\mu)}}\right\} .
\end{aligned}
$$

The energy dependence is entirely contained within the curly brackets which has exactly the same form as Eq. (5) so the sum over the residues leads to exactly the same decay rate as before. The higher order decay is introduced by the subsequent differentiation with respect to $D$. We can then write the following general result. If $C_{m}(\mu)$ vanishes to order $p$,

$$
\left.\Delta \mathcal{E}(\pi) \approx \operatorname{Im} \sum_{m} \frac{d^{p} C_{m}}{d \omega^{p}}\right|_{\omega=\mu} \frac{e^{i Q_{m}(\mu) D}}{2\left(Q_{m}^{\prime}\right)^{p+1} \pi D^{p+1}} .
$$

For the case where $C_{m}(\mu) \neq 0$, then $p=0$ and Eq. (8) reduces to the result previously obtained in Eq. (6). Equation (8) demonstrates clearly that the rate of decay is determined by the leading order term surviving when the Fourier coefficients are expanded about $\omega=\mu$. For each Fourier coefficient to vanish in this fashion, the function itself must vanish at $\omega=\mu$. This requires that $G_{A, B}^{\sigma}=0$, which in turn can only be satisfied for $\Gamma_{a, b}^{(n)}(\mu)=0$. This is the condition for observing short ranged decays in the coupling.

To fully elucidate the conditions under which this might occur, it is useful to consider the different cases shown in Fig. 3: the case where the adatom lies atop a single carbon atom $(n=1)$, at a bridge site connecting a pair of atoms $(n$ 


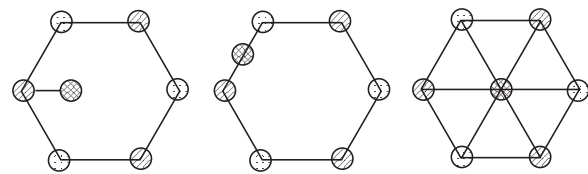

FIG. 3. Three possible impurity locations: atop a single carbon atom, at a bridge site connecting two carbon atoms, and at the center of a hexagon of carbon atoms.

$=2$ ) and finally at the center of a hexagon of carbon atoms $(n=6)$. The stability of transition metal adatoms in each of these positions has been thoroughly examined in. Refs. 12 and 13. The functional form of Eq. (2) is the same in each case; the only difference being the precise form of $\Gamma_{a, b}^{(n)}$ for each of the three values of $n$.

In Fig. 4, we plot $\Gamma_{a, b}^{(n)}(\mu, D)$ as a function of $D$ for the different values of $n$. For a given $n$, there are ${ }^{6} C_{n}$ distinct connections which the adatom can make with surrounding carbon atoms. Most of these cases will not bring about any qualitative difference in behavior. Obviously, for $n=6$, only a single connection can be made. We see from Fig. 4 that $n$ $=6$ is the only value for which the function vanishes for all $D$. To understand why this should be the case, we can employ the analytic expressions for the matrix element between any two propagators on a pristine $(N, N)$ nanotube developed in Ref. 10. It is not necessary to consider the exact quantita-

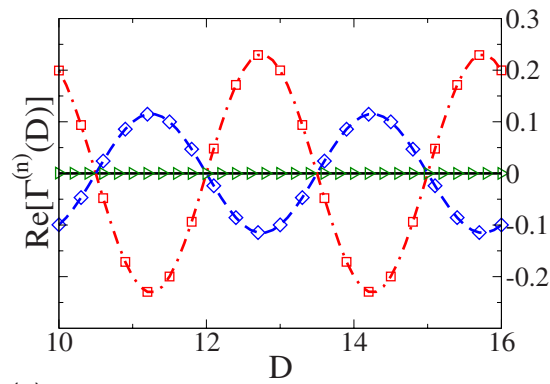

(a)

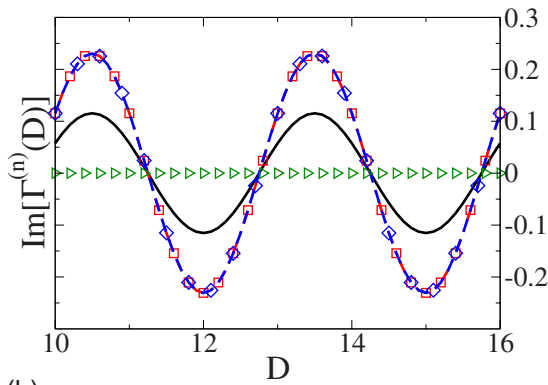

(b)

FIG. 4. (Color online) Real (a) and imaginary (b) parts of $\Gamma^{(n)}$ as a function of $D$ for different values of $n$. The solid line indicates the case of $n=1$. For $n=2$, two inequivalent bridge positions are considered, shown as hatched lines, with the squares and diamonds corresponding to impurities located between atoms $\{1,2\}$ and $\{2,3\}$, respectively (as labeled in Fig. 5). For the case of $n=6$, the data points are represented by triangles. The function is seen to vanish identically only for $n=6$ (see text). In all cases, $\Gamma^{(n)}(D)$ is written in units of inverse nearest neighbor hopping, $\gamma^{-1}$.

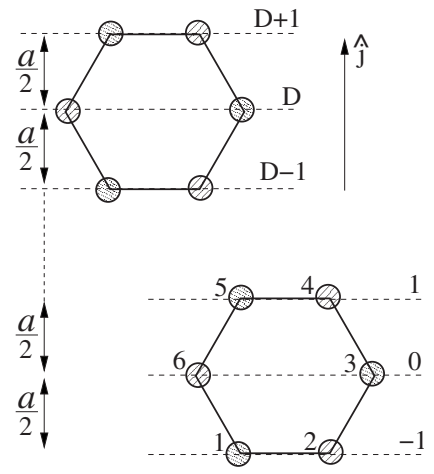

FIG. 5. Two hexagons of carbon atoms whose centers are separated by an amount $D a / 2$ along the tube's longitudinal direction. The atoms are located on six distinct lines labeled $-1,0,1$ and $D$ $-1, D, D+1$. The separation between adjacent planes is $a / 2 \hat{\jmath}$. The hexagon atoms are labeled from 1 to 6 in the anticlockwise direction.

tive expression since its form is somewhat convoluted and we need only make use of the the following salient fact: For a given energy $\omega$, the Green's function will oscillate as a function of $D$, with each nanotube subband contributing a period of oscillation determined by its wave vector $\vec{k}(\omega)$. Around $\omega=\mu=0$, only two subbands, corresponding to electrons propagating along the tube's longitudinal axis, cross the Fermi surface; the contributions from the other modes are strongly attenuated. The periods of these two oscillations are $\pm 4 \pi / 3 a$ at $\mu=0$. Since, as mentioned above, these modes correspond to electrons propagating along the tube axis, the matrix elements will depend only on the longitudinal separation of the adatoms; any separation in the circumferential direction will vanish from the equations.

Furthermore; in an armchair nanotube, the longitudinal separation between any two carbon atoms must be an integer multiple of $a / 2$ (Fig. 5). It follows that if the carbon atoms lie on consecutive lines along this axis, then the sum over the Green function matrix elements will vanish identically since each oscillatory component is phase shifted by an amount $2 \pi / 3$. A necessary condition for this to occur is that $n$ is a multiple of 3 . This is not sufficient for $n=3$ since the atoms must lie on three separate lines. In fact, a further requirement is that the three atoms are located at equivalent points on the underlying graphene sheet (atom labels all even or all odd in Fig. 5). The case of $n=3$ is most likely unphysical but it is useful in understanding why $\Gamma^{(6)}(\mu)$ vanishes. Since the sum over propagators linking any atom in $\mathrm{A}(\mathrm{B})$ with any three atoms on $\mathrm{B}(\mathrm{A})$, subject to each of the atoms on $\mathrm{B}(\mathrm{A})$ being equivalent atoms on separate lines, then the sum over 36 individual matrix elements can be partitioned into 12 separate terms, each of which vanishes. Explicitly $\mathcal{G}_{\ell, 1}+\mathcal{G}_{\ell, 3}$ $+\mathcal{G}_{\ell, 5}=0$ and $\mathcal{G}_{\ell, 2}+\mathcal{G}_{\ell, 4}+\mathcal{G}_{\ell, 6}=0$, where $\ell$ is any atom in hexagon $\mathrm{A}$, and the second index labels atoms in hexagon $\mathrm{B}$ according to the scheme shown in Fig. 5. As a consequence, $\Gamma^{(6)}(\mu)$ vanishes for all $D$. This satisfies the condition for the coupling to decay more rapidly than $1 / D$. How rapidly the coupling, in fact, decays depends on $p$, the leading order term of the Fourier coefficients $C_{m}(\mu)$ surviving in Eq. (7). Since the functions $\Gamma^{(n)}(\omega)$ enter in Eq. (1) as quadratic 

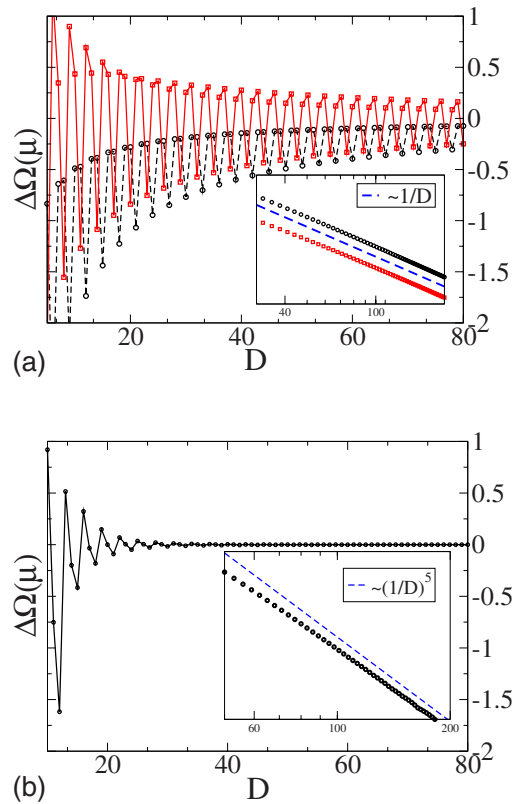

FIG. 6. (Color online) Panel (a) shows the coupling as a function of separation $D$ for the atop (dashed line, circles) and bridge (solid line, squares) positions. A log-log plot of both functions (inset) demonstrates that the coupling decays as $\sim 1 / D$ (hatched line in inset). For clarity, only every third point has been included in the inset. Panel (b) shows the coupling for the center position. The rate of decay is much greater, as evidenced by the inset, which shows the $\log$-log plot with $(1 / D)^{5}$ as a guideline. Note that all couplings are written in arbitrary units.

terms, the minimum value $p$ can have is 2 . However, it is relatively easy to show that $\omega=\mu=0$ is additionally a stationary point of $\Gamma^{(6)}(\omega)$. Therefore, the leading order term in Eq. (7) will be the $p=4$ term.

Figure 6 shows the coupling $\mathcal{E}(\pi)$ as a function of $D$ for each of the three cases considered above. In both graphs, the inset provides a log-log plot of the amplitude of the oscillations. All oscillate with a three atomic layer period, characteristic of armchair nanotubes. The two functions in Fig. 6(a) correspond to the adatoms lying at the atop and bridge positions (dashed and solid lines respectively). As expected, the coupling decays with the standard $1 / D$ dependence predicted for a quasi-one-dimensional host. In Fig. 6(b), the adatoms are located at the center position and the coupling is far more rapidly suppressed. A log-log plot confirms that the decay rate is, in fact, $(1 / D)^{5}$, which is exactly the prediction of Eq. (8) for $p=4$.

First-principles calculations, carried out using the SIESTA package, have been performed on a $(4,4)$ nanotube with two Mn atoms adsorbed on its surface. We adopted a generalized gradient approximation (GGA) approach for the exchange and correlation potentials. ${ }^{14}$ Table I shows the coupling values $[J=\Delta \mathcal{E}(\pi)]$, calculated as the difference in total energy between the ferromagnetic and antiferromagnetic configurations. The calculations were performed for two different values of the adatom separation distance $D$, within a ten ring unit cell. The last column contains the decaying exponent $(\alpha)$ obtained by imposing the condition that the coupling decays according to the law $1 / D^{\alpha}$. These results were obtained for
TABLE I. A $b$ initio results for the coupling $[J=\Delta \mathcal{E}(\pi)]$ and decaying exponent $(\alpha)$ for two Mn atoms adsorbed on the $(4,4)$ nanotube surface separated by a distance $D$. The adatoms are located in the atop and center positions.

\begin{tabular}{lccc}
\hline \hline Position & $\begin{array}{c}D \\
(\end{array}$ & $\begin{array}{c}J \\
(\mathrm{eV})\end{array}$ & $\alpha$ \\
\hline Atop & 4.929 & 0.028 & 2.5 \\
& 7.381 & -0.027 & \\
Center & 4.902 & -0.063 & 4.9 \\
& 7.290 & -0.009 & \\
\hline \hline
\end{tabular}

both atop and center positions. For the case of centrally located atoms, the coupling was found to decay with an exponent $\sim 4.9$, which agrees very satisfactorily with the tightbinding predictions shown in Fig. 6(b). In contrast, $a b$ initio calculations for adatoms located in the atop position returned an exponent $\sim 2.5{ }^{15}$ In presenting these values, the following two points should be made: the SIESTA calculations describe repeating systems of two adatoms in a ten ring unit cell, whereas the tight-binding calculation models an infinite system with two single impurities. The separations involved $(D \leqslant 10 a)$ are far from the asymptotic limit assumed above. Without further simulations, particularly with larger unit cells, it is difficult to be sure that exponents do in reality possess such high values. Nevertheless, the fact that these results agree so closely in with those of our empirical model is remarkable.

In conclusion, by examining the IEC between adatoms located at different positions on a nanotube host, we have confirmed that, although the coupling is indeed in general long ranged, there are cases where it may decay far more abruptly than previously predicted. ${ }^{10}$ Accordingly, the framework provided here, which encompasses both cases, can be considered a generalization of our previous results. ${ }^{10}$ The relative simplicity and transparency of the expressions enable not only the statement of a formal condition underpinning any deviation from the standard behaviour, but also allow a more intuitive picture of why such deviations occur.

Of the three distinct locations where an impurity might realistically be expected to position itself (atop, bridge, center), the center site exhibits by far the most rapid decay $\sim 1 / D^{5}$. This is ultimately understood as resulting from a commensurability between the propagating wave vectors at $\mu=0$ (i.e., $\pm 4 \pi / 3 a$ ) and the number of surrounding atoms. When this number is a multiple of 3 , the contributions from the $3^{2}=9$ (or $6^{2}=36$ in the case of six surrounding atoms) relevant matrix elements can cancel each other out. In this way, the abrupt decay can tell us something about the location of the magnetic impurities. It should perhaps be remarked that such an occurrence might not be entirely coincidental or indeed unique to armchair nanotubes. It may well reflect an intrinsic property of lattices, in general. However, such considerations are outside the scope of the present paper. Finally, it is worth mentioning briefly the important role this effect might play in the rapidly expanding field of chemical sensing. Since the behavior of the coupling depends so dramatically on the position of the adatoms within 
the host, this should, in principle, make it possible to clearly identify the position and nature of the foreign object interaction with the host. Alternatively, our results suggest that by controlling the location where the adsorbed impurities are positioned, we can engineer the range of the magnetic interaction on nanotubes and indirectly manipulate the overall magnetic order of the system. Since temperature variations are known to induce the migration of adsorbed impurities between different locations of the host, this could give another dimension to the selectivity of magnetic properties in low-dimensional systems.

The authors wish to thank Science Foundation Ireland for financial support. One of us (A.T.C.) acknowledges the support of the Brazilian agency $\mathrm{CNPq}$. *ferreirm@tcd.ie

${ }^{1}$ K. Tsukagoshi, B. W. Alphenaar, and H. Ago, Nature (London) 401, 572 (1999).

${ }^{2}$ L. E. Hueso, J. M. Pruneda, V. Ferrari, G. Burnel, J. P. ValdesHerrera, B. D. Simons, P. B. Littlewood, E. Artacho, A. Fert, and N. D. Mathur, Nature (London) 445, 410 (2007).

${ }^{3}$ M. S. Ferreira and S. Sanvito, Phys. Rev. B 69, 035407 (2004).

${ }^{4}$ O. Cespedes, M. S. Ferreira, S. Sanvito, M. Kociak, and J. M. D. Coey, J. Phys.: Condens. Matter 16, 155 (2004).

${ }^{5}$ S. B. Fagan, R. Mota, R. J. Baierle, A. J. R. da Silva, and A. Fazzio, Mater. Charact. 50, 183 (2003).

${ }^{6}$ S. B. Fagan, R. Mota, A. J. R. da Silva, and A. Fazzio, Phys. Rev. B 67, 205414 (2003).

${ }^{7}$ S. B. Fagan, R. Mota, R. J. Baierle, A. J. R. da Silva, and A. Fazzio, Physica B 340, 982 (2003).

${ }^{8}$ C. K. Yang, J. Zhao, and J. P. Lu, Phys. Rev. Lett. 90, 257203 (2003).
${ }^{9}$ Yuchen Ma, A. S. Foster, A. V. Krasheninnikov, and R. M. Nieminen, Phys. Rev. B 72, 205416 (2005).

${ }^{10}$ A. T. Costa, D. F. Kirwan, and M. S. Ferreira, Phys. Rev. B 72, $085402(2005)$.

${ }^{11}$ A. T. Costa, C. G. Rocha, and M. S. Ferreira, Phys. Rev. B 76, 085401 (2007).

${ }^{12}$ Y. Yagi, T. M. Briere, M. H. F. Sluiter, V. Kumar, A. A. Farajian, and Y. Kawazoe, Phys. Rev. B 69, 075414 (2004).

${ }^{13}$ S. B. Fagan, R. Mota, A. J. R. da Silva, and A. Fazzio, J. Phys.: Condens. Matter 16, 3647 (2004).

${ }^{14}$ J. P. Perdew, K. Burke, and M. Ernzerhof, Phys. Rev. Lett. 77, 3865 (1996).

${ }^{15}$ The discrepancy with the predicted decaying exponent of 1 is explained by the fact that this is an asymptotic rate of decay. Improvements in the comparison would require an extremely large unit cell in the $a b$ initio calculation. 